\title{
Horizontal transfer of transposons between and within crustaceans and insects
}

\author{
Mathilde Dupeyron', Sébastien Leclercq ${ }^{1}$, Nicolas Cerveau ${ }^{1,2}$, Didier Bouchon ${ }^{1}$ and Clément Gilbert ${ }^{1 *}$
}

\begin{abstract}
Background: Horizontal transfer of transposable elements $(H T)$ is increasingly appreciated as an important source of genome and species evolution in eukaryotes. However, our understanding of HTT dynamics is still poor in eukaryotes because the diversity of species for which whole genome sequences are available is biased and does not reflect the global eukaryote diversity.

Results: In this study we characterized two Mariner transposable elements (TEs) in the genome of several terrestrial crustacean isopods, a group of animals particularly underrepresented in genome databases. The two elements have a patchy distribution in the arthropod tree and they are highly similar (>93\% over the entire length of the element) to insect TEs (Diptera and Hymenoptera), some of which were previously described in Ceratitis rosa (Crmar2) and Drosophila biarmipes (Mariner-5_Dbi). In addition, phylogenetic analyses and comparisons of TE versus orthologous gene distances at various phylogenetic levels revealed that the taxonomic distribution of the two elements is incompatible with vertical inheritance.

Conclusions: We conclude that the two Mariner TEs each underwent at least three HTT events. Both elements were transferred once between isopod crustaceans and insects and at least once between isopod crustacean species. Crmar2 was also transferred between tephritid and drosophilid flies and Mariner-5 underwent HT between hymenopterans and dipterans. We demonstrate that these various HTTs took place recently (most likely within the last 3 million years), and propose iridoviruses and/or Wolbachia endosymbionts as potential vectors of these transfers.
\end{abstract}

Keywords: Horizontal transfer, Transposable elements, Isopod crustaceans, Hexapods

\section{Background}

Horizontal transfer $(\mathrm{HT})$ of genetic material is the transmission of DNA between non-mating organisms [1]. Most known eukaryote-to-eukaryote HT events are transfers of transposable elements (TEs) [2]. Given the profound impact TEs have on the genome architecture of their hosts, HT of TEs (HTT) is increasingly recognized as an important force in eukaryote genome evolution [3]. On the TE side, spreading between genomes via HT may be viewed as a strategy to escape vertical extinction due to purifying selection, mutational decay and/or host defense mechanisms. Among the over 330 cases of eukaryote-to-eukaryote HTT events characterized so far, the vast majority involve DNA transposons

\footnotetext{
* Correspondence: clement.gilbert@univ-poitiers.fr

'Université de Poitiers, UMR CNRS 7267 Ecologie et Biologie des Interactions, Equipe Ecologie Evolution Symbiose, 86022 Poitiers, Cedex, France

Full list of author information is available at the end of the article
}

( $\mathrm{n}=188$ cases) and LTR retrotransposons ( $\mathrm{n}=118$ cases) [4], indicating that the long-term survival of these TEs may rely more on HT than that of non-LTR retrotransposons. Yet, while whole genomes are sequenced at an exponential pace, the global diversity of eukaryote genomes is still poorly represented, precluding any strong generalization on HTT dynamics. Even in animals, whole genome sequencing efforts are biased towards species closely related to model organisms or species of economic interest, and whole genome sequences are lacking for many large taxonomic groups. Our current understanding of the global HTT dynamics and impacts is therefore incomplete, both at the host and TE level.

With only one genome fully sequenced [5] out of over 50,000 species described [6], crustaceans are particularly

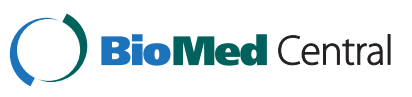

(c) 2014 Dupeyron et al.; licensee BioMed Central Ltd. This is an Open Access article distributed under the terms of the Creative Commons Attribution License (http://creativecommons.org/licenses/by/2.0), which permits unrestricted use, distribution, and reproduction in any medium, provided the original work is properly credited. The Creative Commons Public Domain Dedication waiver (http://creativecommons.org/publicdomain/zero/1.0/) applies to the data made available in this article, unless otherwise stated. 
underrepresented in genome databases. The order Isopoda (Vericrustacea clade according to [7]) is unique among crustaceans in that the colonization of landmasses by one of its lineages (belonging to the suborder Oniscidea) during the Mesozoic yielded a large diversity of terrestrial species $(>3,600$ [8]) now distributed all over the world in every biotope (except for the poles) [9]. In this study we report new cases of HTT involving terrestrial isopod crustaceans and hexapods. We used a combination of crossspecies PCR screening of TEs, phylogenetic and other evolutionary analyses to characterize in detail these HTT and to shed light on the evolutionary dynamics of the first two TEs described in isopod crustaceans.

\section{Results and discussion}

\section{Characterization of two Mariner elements in the isopod crustacean Armadillidium vulgare}

In order to detect TEs that underwent horizontal transfer between isopod crustaceans and other taxa, we used all consensus sequences deposited in Repbase [10] as of May 2013 as queries to perform BLASTn searches on draft genomic contigs and on a transcriptome of the pill bug Armadillidium vulgare that have been generated in our lab as part of other ongoing projects. Importantly, the contigs generated by these projects are too short to carry out a comprehensive de novo mining of $A$. vulgare TEs. The BLASTn searches yielded two TEs belonging to the Tc1/Mariner superfamily of Class II DNA transposons that show more than $90 \%$ identity over more than $500 \mathrm{bp}$ to $A$. vulgare sequences. The first one (Crmar2) was originally characterized in the tephritid fly Ceratitis rosa based on a PCR/sequencing screening [11], and the second one (Mariner-5_Dbi) was described by Kojima and Jurka [12] in Drosophila biarmipes based on whole genome sequence data mining. We reconstructed an $A$. vulgare consensus sequence of both elements (named Crmar2_Avul and Mariner-5_Avul) using 100 to $1300 \mathrm{bp}$-long fragments resulting from our various BLAST outputs, such that the entire sequence of the consensus was covered by at least five different copies. Crmar2_Avul is 1304 bp in length, has 39-bp terminal inverted repeats (TIRs) and encodes a 361 amino acid (aa) transposase while Mariner-5_Avul is 1013 bp in length, has 28-bp TIRs and encodes a 200 aa transposase. Both elements are flanked by TA target site duplications, which is characteristic of the Tc1/Mariner superfamily [13]. The evolution of this superfamily has yielded a large number of elements which have colonized the genome of many eukaryote taxa [14,15] and have been classified in various subfamilies (for example, [16]). Crmar2 belongs to the rosa subfamily [11] and our phylogenetic analysis of the transposase revealed that Mariner-5_Dbi belongs to the irritans subfamily [see Additional file 1: Figure S1].

\section{Taxonomic distribution of the two Mariner elements in eukaryotes}

Next we sought to assess the taxonomic distribution of Crmar2_Avul and Mariner-5_Avul in eukaryotes by performing BLASTn searches on all eukaryotic genomes that were available in Genbank as of May 2013. In addition to the species in which the two elements had previously been described (C. rosa, Anastrepha ludens and Anastrepha suspensa for Crmar2; D. biarmipes for Mariner-5_Dbi) we found TEs highly similar (>90\% identity over $>500 \mathrm{bp}$ ) to Crmar2_Avul in Drosophila ananassae and Drosophila bipectinata, and to Mariner5_Avul in the ant Harpegnathos saltator. Interestingly, the taxonomic distribution of the two elements is patchy, not only at the level of the arthropod phylogeny, but also within the lower level taxa in which we found them (Figure 1), a pattern likely indicative of horizontal transfer [17]. For example, Crmar2_Avul is only present in two closely related Drosophila species out of the 13 that we searched, and Mariner-5_Avul was identified only in one of the three hymenopteran genomes available.

\section{Horizontal transfer of the two Mariner elements between hexapods and isopods and within hexapods}

To formally assess whether the distribution of the two elements in arthropods is the result of HT, we compared TE genetic distances calculated between hexapod species and $A$. vulgare to distances calculated for 46 orthologous genes available for both $A$. vulgare and Drosophila melanogaster [7]. As illustrated in Figure 2, distances between orthologous genes (average $=35 \%$, $\min =21 \%$, and $\max =49 \%)$ are much higher than distances between TEs (average $=6 \%$, $\min =4.9 \%$, and $\max =7.4 \%$ ). Under vertical transmission of the TEs, TE distances between taxa are expected to be higher than distances between orthologous genes because TEs are known to evolve neutrally after insertion in a given genome [22], that is, faster than host genes that evolve under purifying selection due to functional constraints. The high similarity between $A$. vulgare and hexapoda TEs coupled with the deep divergence time between these two arthropod taxa ( $>400$ million years) and to the patchy distribution of the two elements in arthropods allows us to confidently conclude that the presence of both Crmar2 and Mariner-5 in isopod crustaceans and hexapods results from HT. Interestingly, we found that gene distances between tephritid (Ceratitis capitata) and drosophilid (D. melanogaster) flies on one hand (average $=27 \%, \min =16 \%$, and $\max =45 \%$ ) and between Drosophila and the ant $H$. saltator on the other hand (average $=34 \%, \min =18 \%$, and $\max =56 \%$ ) are also much higher than TE distances (4\% for both elements; Figure 2). This pattern suggests that in addition to transferring horizontally between hexapods and isopods, Crmar2 also underwent HT 


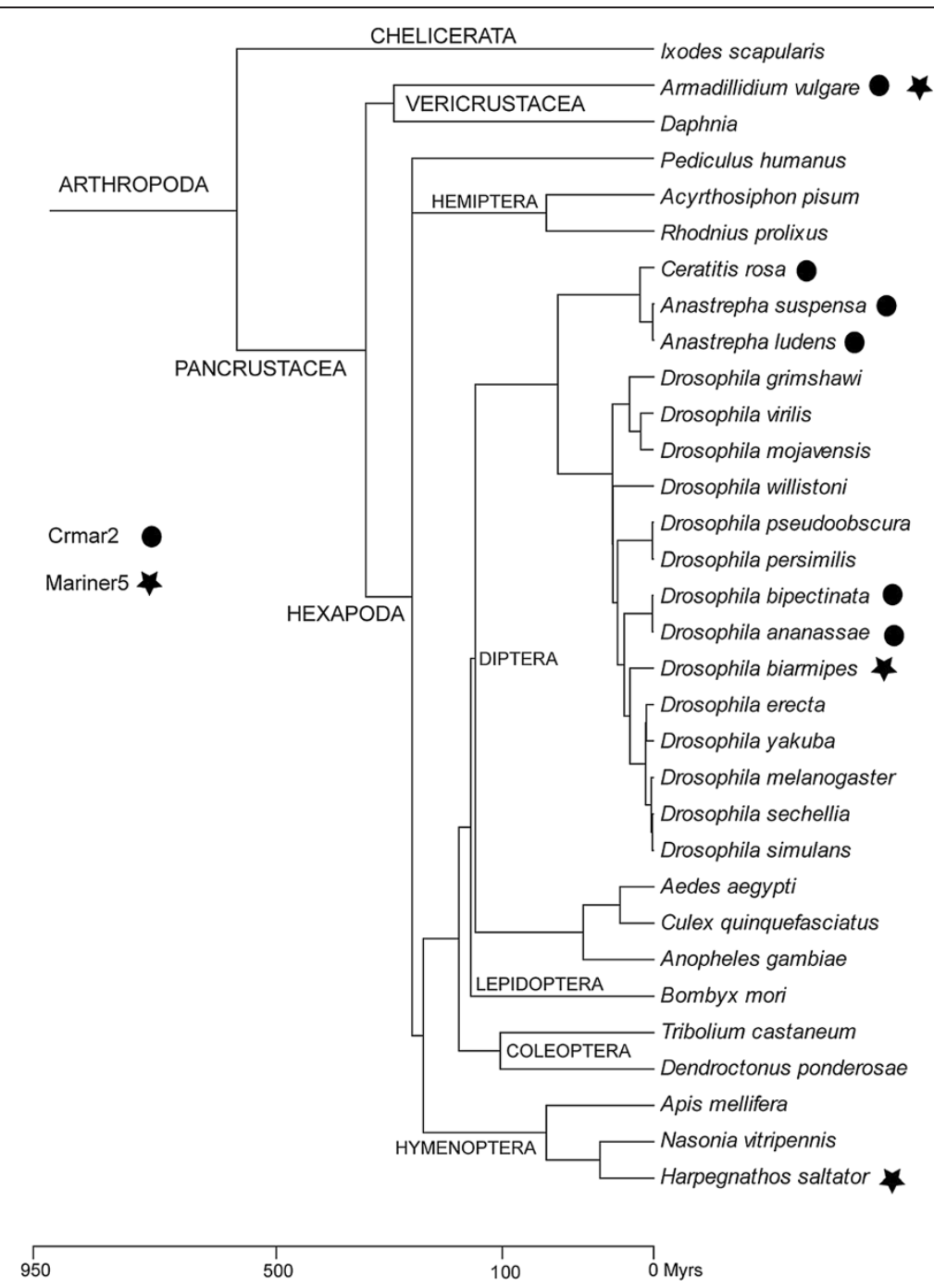

Figure 1 Timetree of Arthropoda. The tree includes all species in which Crmar2 and Mariner- 5 were found as well as most closely related species for which whole genome sequences are available in Genbank. Phylogenetic relationships are taken from Tamura et al. [18], Regier et al. [19], Sharkey [20] and Regier et al. [7]. Divergence times are taken from Hedges et al. [21]. Divergence times between D. bipectinata and D. ananassae, between D. biarmipes and other Drosophila species, between Dendroctonus ponderosae and Tribolium castaneum, between A. vulgare and Daphnia pulex, and between $\mathrm{H}$. saltator and Nasonia vitripennis are unknown and have therefore been set at arbitrary values for illustrative purposes.

between the two dipteran lineages and Mariner-5 also transferred horizontally between dipterans and hymenopterans.

\section{Recent horizontal transfer of the two Mariner elements within isopods}

To shed light on the evolutionary dynamics of Crmar2_Avul and Mariner-5_Avul in terrestrial isopod crustaceans we carried out two sets of PCR screenings in 14 species. The first screening involved primer pairs designed in the internal region of the elements in order to check for the presence of each TE in the various species (type 1 primers in Additional file 2: Figure S2). The second screening aimed at finding specific copies of the two elements that would be shared at orthologous loci between the various isopod species. For the latter screen we used primer pairs for which one primer was designed in the 5' or 3' end of Crmar2_Avul and Mariner-5_Avul and the other primer was designed in the flanking region of several copies of each element (type 2 primers in Additional file 2: Figure S2; $\mathrm{n}=4$ for Crmar2_Avul and 3 for Mariner-5_Avul). The first screening (internal primers) uncovered Crmar2_Avul and Mariner-5_Avul, respectively, in six and nine of the 14 species (Figure 3). The second screening (search for orthologous copies) did not reveal any shared copies between $A$. vulgare and any of the other 13 isopod species. This absence of amplification could be due to a lack of conservation of the regions flanking the various copies in the different 


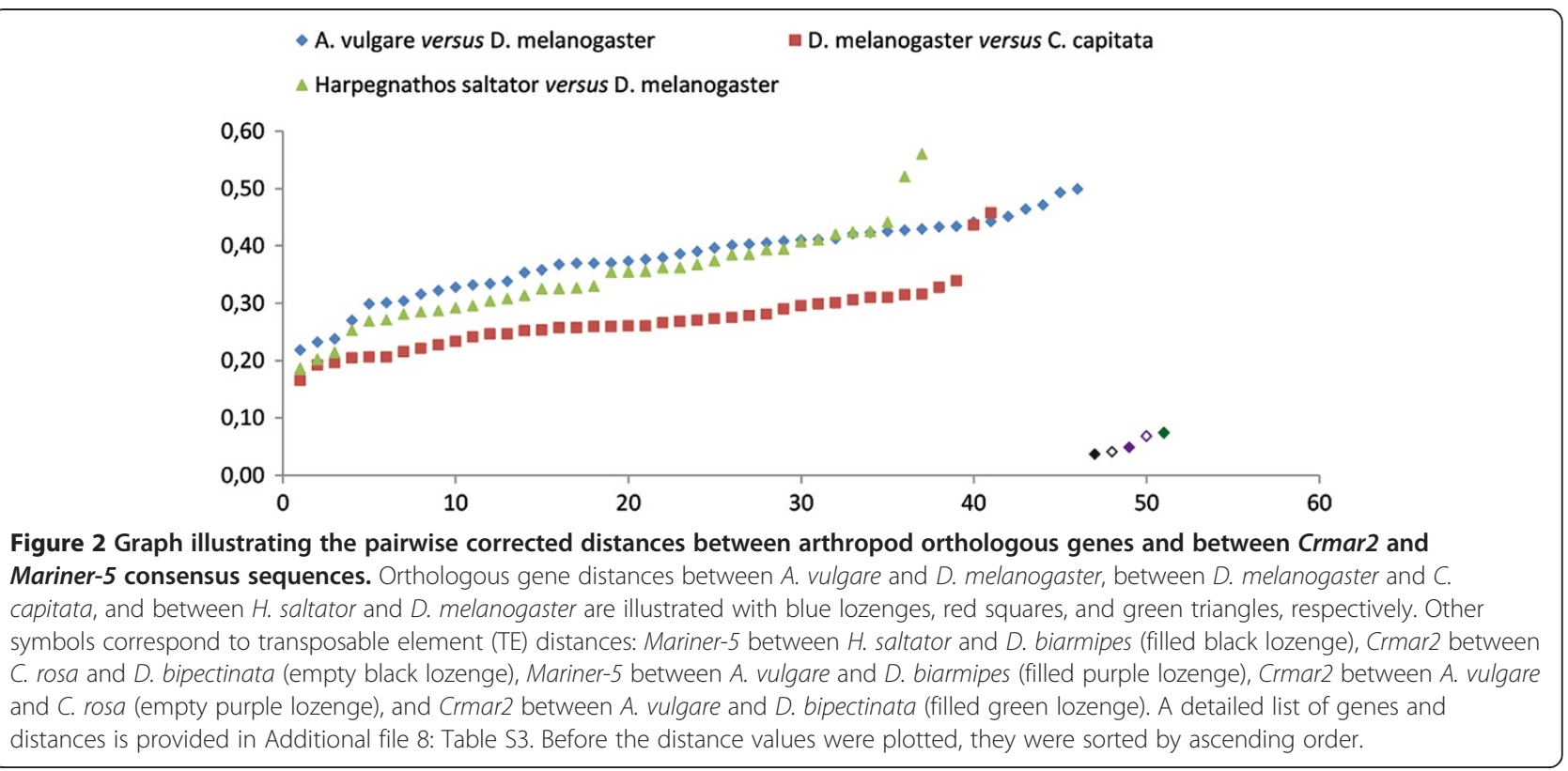

species. But perhaps more interestingly, we also noticed that two of the four Crmar2 copies failed to amplify in one of the two $A$. vulgare individuals in which we searched them, suggesting that they are polymorphic in terms of presence/absence in A. vulgare populations. Overall, these data indicate that both elements likely underwent recent HT in isopods and may still be active.
To further test the possibility that both elements invaded isopod genomes recently via $\mathrm{HT}$ and are still actively transposing, we cloned and sequenced two to five different copies of Crmar2_Avul and Mariner-5_Avul in all isopod species in which we found them (except for Eluma purpurascens in which all ten clones that we sequenced contained an identical copy of Crmar2).

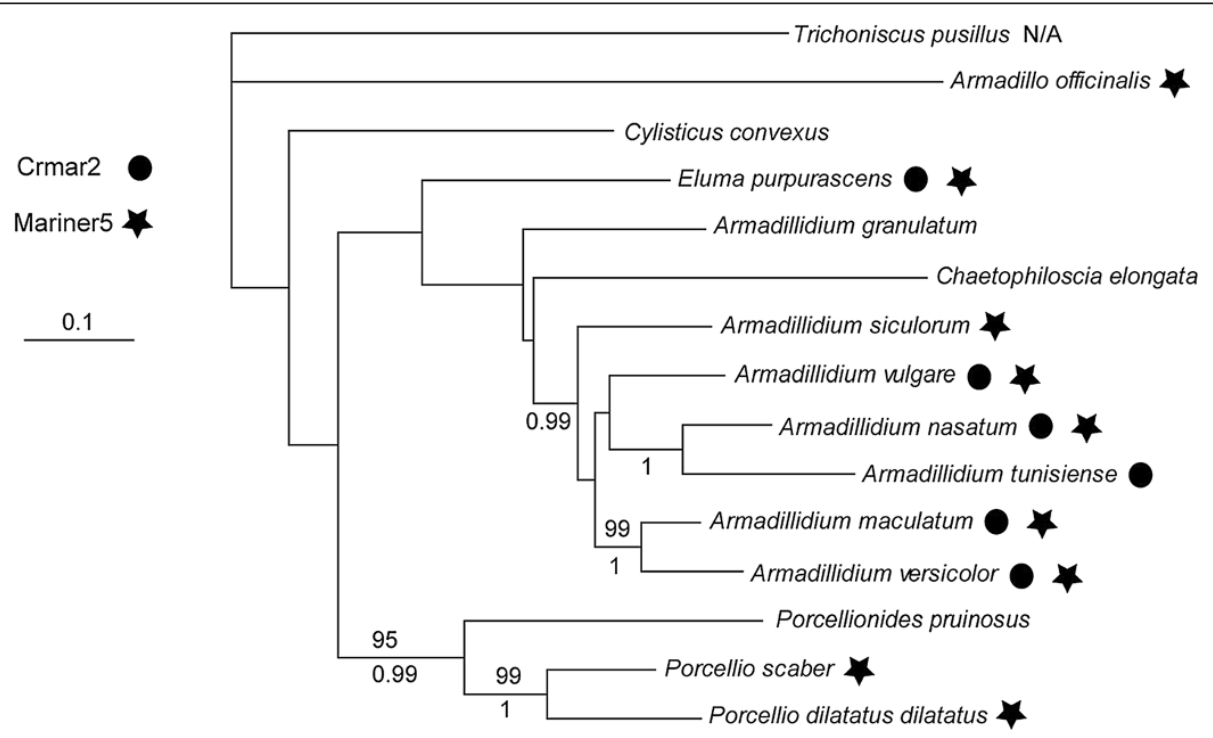

Figure 3 Phylogenetic tree of the various terrestrial isopod crustacean species included in this study. The topology of the tree corresponds to the consensus of the bootstrap analysis performed under the maximum likelihood criteria. Bootstrap values above $70 \%$ and Bayesian posterior probabilities above 0.9 are shown above and below branches respectively. Species in which Crmar2 and/or Mariner-5 were uncovered by PCR are marked with a filled circle and/or a star. Trichoniscus pusillus was used as an outgroup to root the present phylogeny based on the topology obtained in Michel-Salzat and Bouchon [23] and was not screened for the presence of the two TEs (N/A: not applicable). The other species were PCR screened but none of the two elements were amplified. 
Pairwise genetic distances between these copies within each genome are all very low (average $=1.2 \%, \min =$ $0.9 \%$, and $\max =2 \%$ for Crmar2_Avul and average $=$ $4.5 \%$, $\min =2 \%$, and $\max =6 \%$ for Mariner-5_Avul). In addition, the between-species distances for both TEs are also much lower than distances calculated for the androgenic gland hormone gene (average $=34 \%$; Figure 4 ). Following the same reasoning as for the comparisons between TE and orthologous gene distances discussed above, we believe these results strongly suggest that both Crmar2_Avul and Mariner-5_Avul underwent one or more recent HTs within isopods. Interestingly, using RTPCR, we verified that Crmar2_Avul and Mariner-5_Avul are transcribed both in somatic and germ cells in $A$. vulgare [see Additional file 3: Figure S3]. Furthermore, seven of the eight Crmar2_Avul transcripts that we uncovered in the A. vulgare transcriptome contain a fulllength and intact (devoid of non-sense mutations) ORF (sequences provided in Additional file 4: Dataset 1), suggesting that at least one source of functional transposase is transcribed for this element in A. vulgare.

To provide an estimate of the absolute age of the activity burst of Mariner-5 and Crmar2, we divided the average copy/consensus distance calculated for each element in D. biarmipes (11\%) and D. bipectinata (6.9\%) by the experimentally derived neutral substitution rate of D. melanogaster (0.0346 substitutions per base per million years (myr); [24-26]). This yielded a burst age of 3.2 myrs for Mariner-5 in D. biarmipes and 2 myrs for Crmar2 in D. bipectinata. The age of Mariner-5_Avul and Crmar2_Avul cannot be precisely estimated because nuclear substitution rates are not available for isopods. Together with the absence of shared orthologous copies of both elements between the various isopods species and the seemingly polymorphic state of Crmar2 in A. vulgare, the fact that an intact Crmar2_Avul transposase is transcribed in this species is consistent with a recent invasion of isopod genomes by Mariner-5_Avul and Crmar2_Avul and suggest both elements are active sources of genomic variation in this major crustacean group. In addition, given that isopod Crmar2 and Mariner-5 are highly similar to Drosophila Crmar2 and Mariner-5 (95\% and $93 \%$ identity, respectively; Figure 2), we believe these HTTs most likely took place within the past few million years at most.

\section{Number of horizontal transfer of transposon events}

To assess the number of HTTs that occurred between hexapods and crustacean isopods and within each of the two taxa, we reconstructed a phylogeny of both elements based on an alignment including all copies of Crmar2 and Mariner-5 that we sequenced from the various isopod species and those that we found in the other sequenced arthropod genomes. In the resulting Crmar2 tree both hexapod and isopod Crmar2 elements are monophyletic (Figure 5). Therefore, a single HTT event between isopods and hexapods needs to be inferred to explain the taxonomic distribution of this element in arthropods. Within hexapods, Crmar2 TEs from the tephritid C. rosa are more closely related to Drosophila elements than they are to the other tephritid elements found in A. ludens and A. suspensa by Gomulski et al. [11]. This topology upholds our TE versus orthologous gene distance analysis (see above), indicating that Crmar2 also underwent HT within dipterans. In the Mariner-5 tree (Figure 6), isopod elements fall within

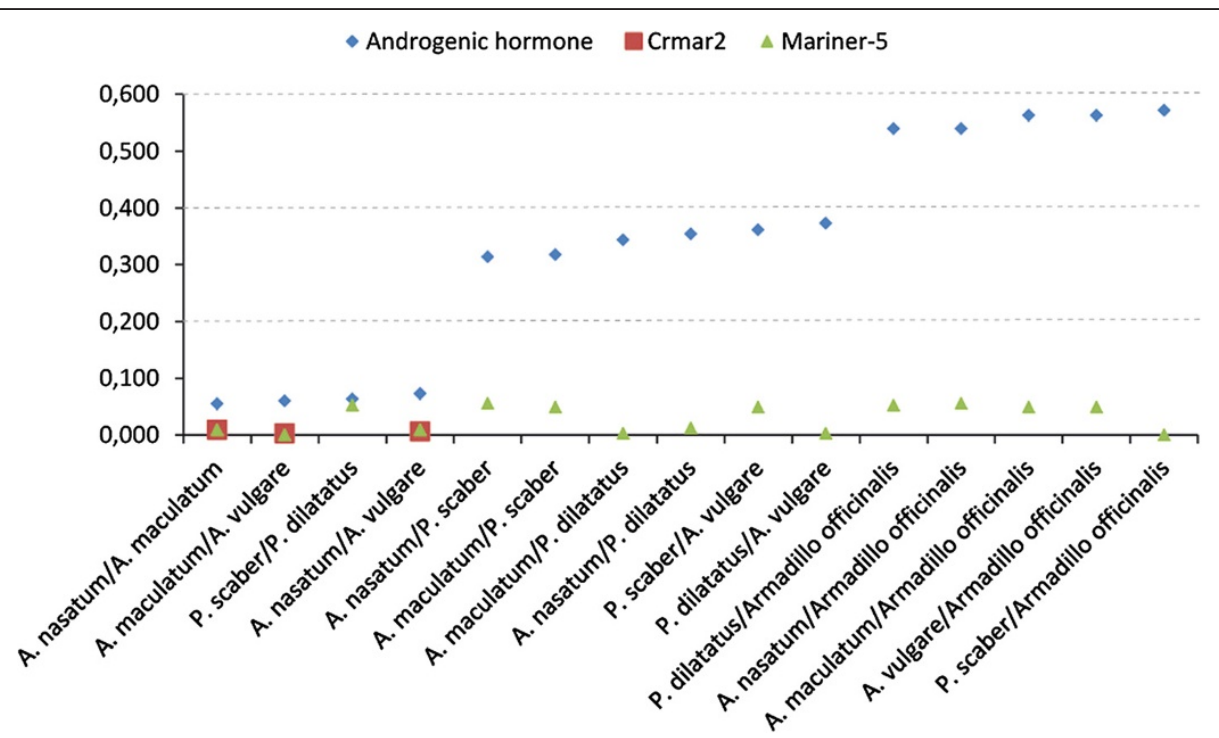

Figure 4 Graph illustrating the pairwise corrected distances between the androgenic gland hormone gene and between Crmar2 and Mariner-5 consensus sequences within terrestrial isopods. 


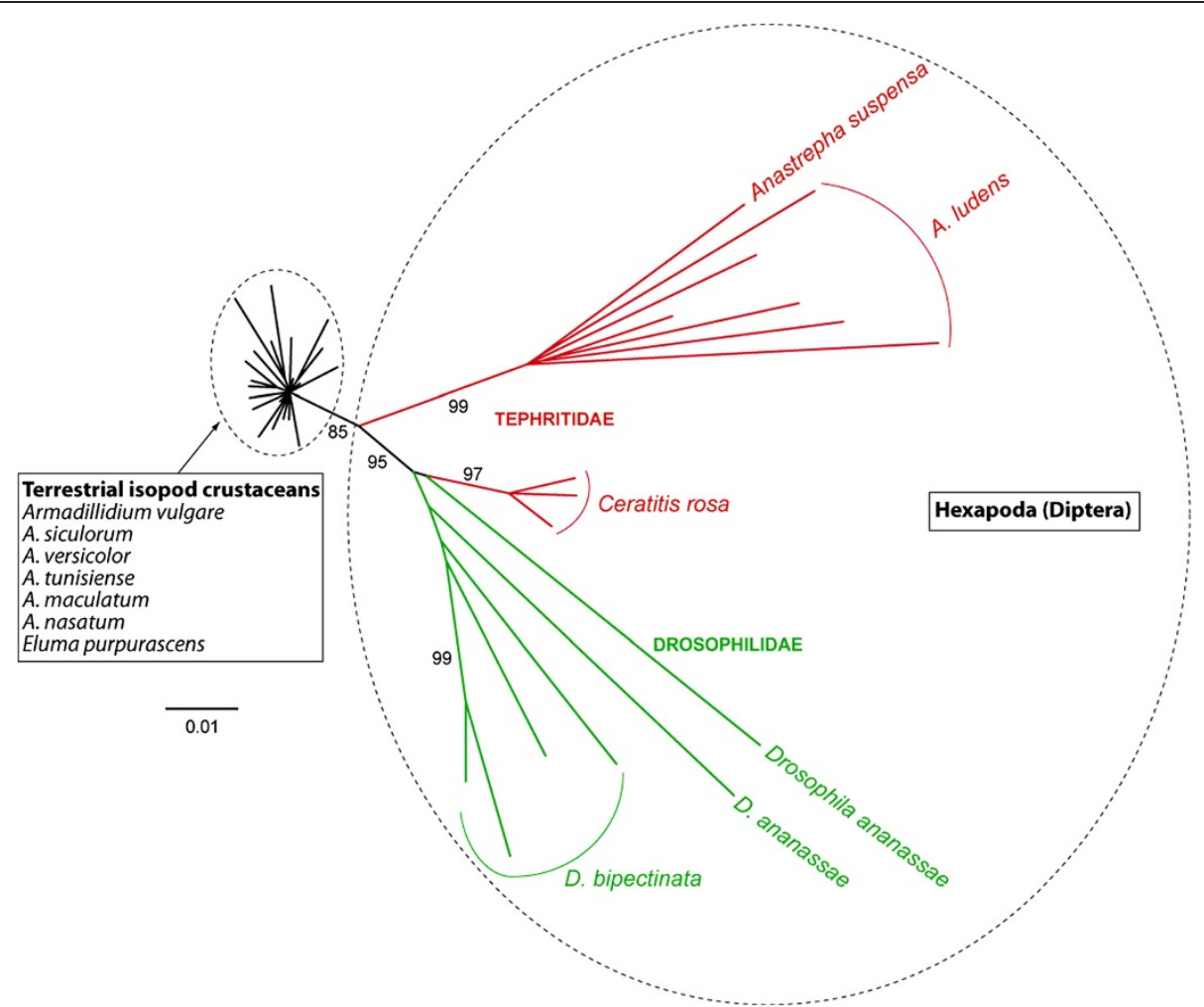

Figure 5 Phylogenetic tree of Crmar2 copies. Maximum likelihood bootstrap values above $70 \%$ are shown on branches. Given the absence of phylogenetic support for the branching of Crmar2 copies within isopods, the name of the species is shown only once in the black rectangle to facilitate the reading of the figure.

two relatively distantly related clusters. However, given that the tree is unrooted, we cannot conclude on whether Mariner-5 was transferred once or more than once between hexapods and isopods. Interestingly, the topology of isopod Mariner-5 copies (Figure 6) is clearly incongruent with that of the isopod tree (Figure 3). For example, Porcellio dilatatus dilatatus and Porcellio scaber form a strongly supported clade in the species phylogeny, but Crmar2 copies from the former fall within the cluster that groups all Armadillidium and Eluma purpurascens copies and those of $P$. scaber group with Crmar2 copies from Armadillo officinalis. This phylogenetic incongruence between host and TE phylogenies, together with the general lack of phylogenetic resolution within isopod Crmar2 and Mariner-5 clusters (Figures 5 and 6), and the fact that copies from each isopod species do not form monophyletic groups, further supports the HT of both elements between the various isopod species.

\section{Potential vectors of horizontal transfer}

Though the question of the mechanisms and vectors underlying HTT between multicellular eukaryotes remains largely open, growing evidence suggests that hostparasite relationships likely facilitate such transfers
[27-31]. In particular, viruses are often cited as ideal HTT vectors due to their capacity to inject DNA/RNA into host cells [32-35]. Though the viral fauna infecting the various species involved in Crmar2 and Mariner-5 HTT is poorly known, it is noteworthy that members of the Iridoviridae have been found in several species of dipterans, hymenopterans and terrestrial isopods [36,37]. In addition, a recent study identified two TEs inserted in the genome of an iridovirus infecting dipteran [38], emphasizing the potential of this type of viruses to shuttle transposons between their hosts. Another possible route for HTT to occur in arthropods is via transfers of endosymbiotic bacteria. Several species of isopods as well as $D$. ananassae, $D$. bipectinata and $A$. suspensa are known to bear intracellular, maternally transmitted alphaproteobacteria called Wolbachia [39-43]. The fact that isopod Wolbachia strains are known to have undergone several HT [44] and that several genes of eukaryotic origin have been found integrated in Wolbachia genomes [45-47] suggest that endosymbionts could also facilitate HT of DNA between hosts.

\section{Conclusions}

In this study, we have characterized the evolutionary dynamics of two Tc1/Mariner elements in isopod crustaceans 


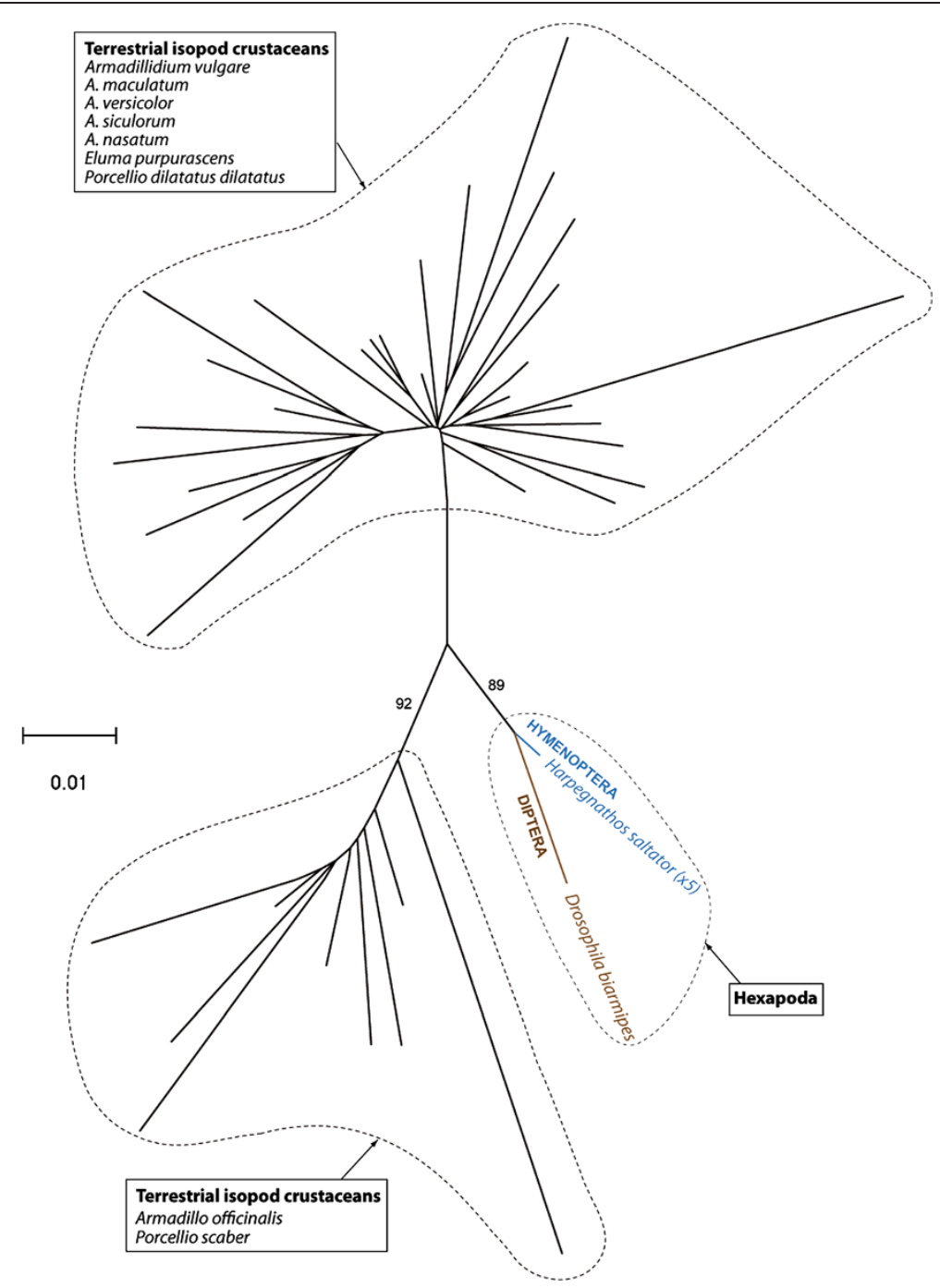

Figure 6 Phylogenetic tree of Mariner-5 copies. Maximum likelihood bootstrap values above 70\% are shown on branches. Given the absence of phylogenetic support for the branching of Mariner-5 copies within the two clusters of isopod sequences, the name of the species is shown only once in the two black rectangles to facilitate the reading of the figure.

and shown that their current taxonomic distribution in arthropods results from at least one HT between hexapods and isopods as well as one or more HTs within isopods. Furthermore, we have demonstrated that Crmar2 transferred horizontally between drosophilid and tephritid flies and that Mariner-5 underwent HT between Diptera and Hymenoptera. Conservatively, and assuming that Crmar2 and Mariner-5 transferred horizontally only once within the isopods, we have uncovered a total of six new HTT events in this study. Together with 70 previously known cases (for example, [48-50]; reviewed in [4]) our results bring to 76 the number of HT events described in metazoans for the Tc1/Mariner superfamily, further emphasizing the indifference of these elements to host factors to transpose [51]. Of note, HT of two other Mariner elements have previously been characterized in marine crustaceans [52] (one between two decapods and one between a decapod and an amphipod), but our study is the first to report HTT involving terrestrial crustaceans. Finally, we have shown that these newly described HTT events most likely took place within the last 3 myrs, and we propose iridoviruses and/or Wolbachia endosymbionts as the potential vectors of transfer, a hypothesis that will be interesting to test in future studies.

\section{Methods}

Mining of available eukaryote genomes

In order to identify transposable elements similar to Crmar2 and Mariner-5_Dbi that could have been horizontally transferred between isopods and other taxa we used the nucleotide sequence of the two elements to carry out BLASTn searches against the nr (non-redundant nucleotide), EST (expressed sequence tag) and 
WGS (whole genome sequence) databases available on the NCBI website. We considered only those elements that showed more than $90 \%$ nucleotide identity over more than $500 \mathrm{bp}$ of our query sequences.

\section{DNA extraction, PCR, cloning, and sequencing}

Genomic DNA was extracted from 14 species of terrestrial isopods (Figure 3) using the Qiagen ${ }^{\text {th }}$ DNeasy blood and tissue extraction kit (Hilden, Germany). PCRs were carried out using four types of primer pairs: 1) one pair designed on the internal region of Mariner-5_Avul and Crmar2_Avul, 2) three and four pairs designed to screen specific copies of the two elements at orthologous position in the 14 isopod species, 3) one pair designed to amplify the mitochondrial cytochrome oxidase I (Col) for the six species for which this gene is not available in Genbank, and 4) one pair designed to amplify the mitochondrial $16 \mathrm{~S}$ gene for all species included in this study except for T. pusillus (taken from Genbank). The list of PCR primers used in this study, together with their respective melting temperatures, is given in Additional file 5: Table S1. PCR reactions were conducted using the following temperature cycling: initial denaturation at $94^{\circ} \mathrm{C}$ for $5 \mathrm{~min}$, followed by 30 cycles of denaturation at $94^{\circ} \mathrm{C}$ for $30 \mathrm{~s}$, annealing at 52 to $58^{\circ} \mathrm{C}$ (depending on the primer pair) for $30 \mathrm{~s}$, and elongation at $72^{\circ} \mathrm{C}$ for $45 \mathrm{sec}$, ending with a $10 \mathrm{~min}$ elongation step at $72^{\circ} \mathrm{C}$. PCR products obtained with the $\mathrm{Co} 1$ primers were purified and directly sequenced using $\mathrm{ABI}$ BigDye sequencing mix (1.4 $\mu \mathrm{l}$ template PCR product, $0.4 \mu \mathrm{l}$ BigDye, $2 \mu \mathrm{l}$ manufacturer supplied buffer, $0.3 \mu \mathrm{l}$ primer, and $6 \mu \mathrm{l} 2 \mathrm{O}$ ). Sequencing reactions were ethanol precipitated and run on an $\mathrm{ABI}$ 3730 sequencer. PCR products obtained with the primers internal to Crmar2_Avul and Mariner-5_Avul were cloned into pGEM-T easy vector (Promega, USA, Madison, WI) and several clones were Sanger-sequenced as described above until we obtained five different copies of each element in the various species in which we found them.

\section{Sequencing of androgenic gland hormone (Agh) CDNA}

Total RNA was isolated from androgenic glands of fifteen males ( 6 glands per individual) using the RNeasy Mini kit (Qiagen, Hilden, Germany). The cDNA was synthesized using the M-MLV-RT kit (Promega). PCR amplification was performed using several degenerated primer pairs designed on the consensus sequences of Agh cDNAs of A. vulgare, P. scaber and P.dilatatus [see Additional file 6: Table S2] [53]. PCR and direct sequencing were performed as described above.

\section{RT-PCR}

The expression of Crmar2_Avul and Mariner-5_Avul was assessed in both dissected ovaries and somatic tissues (head + nervous chain) of $A$. vulgare females using the SuperScript ${ }^{\mathrm{ma}}$ III First-Strand Synthesis System for RT-PCR» (Invitrogen, Eugene, OR, USA).

\section{Transposon distances versus gene distances}

All Crmar2 and Mariner-5 consensus sequences reconstructed in this study are provided in Additional file 7: Dataset 2. In order to test whether Crmar2 and Mariner-5 TEs were inherited vertically or horizontally we compared the distances calculated between TE consensus sequences and between several orthologous genes for several pairs of taxa. To calculate gene distances between Isopoda and Hexapoda, we used the 57 A. vulgare genes sequenced by Regier et al. [7] as queries to perform BLASTn searches against the $D$. melanogaster genome. We chose the D. melanogaster genome rather than the genome of Drosophila species involved in the HTT characterized in this study because it is the most completely sequenced and best assembled Drosophila genome available. We found $46 \mathrm{~A}$. vulgare orthologs in $D$. melanogaster, which we aligned at the nucleotide level between the two species. For the Ceratitis/Drosophila gene distances we used the 46 genes resulting from the above search as queries to perform BLASTn searches against the whole genome sequence of $C$. capitata. This search yielded 41 genes that we aligned at the nucleotide level with those of $D$. melanogaster. The same approach was used to find genes orthologous between $D$. melanogaster and $\mathrm{H}$. saltator which resulted in the alignment of 37 genes. Genetic distances between Crmar2 and Mariner-5 consensus sequences as well as between each pair of orthologous genes were calculated using the Jukes Cantor model in MEGA 5 [54]. The name of all genes and the distances between them are provided in Additional file 8: Table S3. Jukes Cantor distances were also calculated between the various copies of both TEs found within each genome in which we found them.

\section{Phylogenetic analyses}

The phylogeny of the 14 terrestrial isopod species understudy was reconstructed using $16 S, C o 1$, and Agh sequences. All sequences produced in this study were deposited in Genbank under accession numbers KF957774-KF957833. Each gene was aligned manually using BioEdit 7.0.5.3 [55], and ambiguous regions were removed. All alignments are provided in Additional file 9: Dataset 3. A bootstrapped neighbor joining phylogeny was first reconstructed using MEGA 5 for each alignment with the maximum likelihood distance option. Given that no incongruence supported by bootstrap values $>50 \%$ was observed between the three resulting trees (not shown), we then concatenated the three alignments and reconstructed a bootstrapped maximum likelihood phylogeny of the three combined markers using PhyML 3 [56]. A bayesian analysis of this alignment was 
also performed using MrBayes [57] in order to obtain posterior probabilities for each node of the tree. The model of nucleotide evolution best fitting the combined alignment $(G T R+I+G)$ and used for the phylogenetic analyses was chosen based on the Akaike information criterion (AIC) in jModeltest 2 [58].

The phylogeny of Crmar2 and Mariner-5 was reconstructed based on alignments of all different copies of each element from each species in which we found them. Ambiguous regions and regions absent in more than $25 \%$ of the sequences were removed. Alignments are provided in Additional file 9: Dataset 3. The model of nucleotide evolution best fitting each alignment (TPM1uf + G for both elements) was chosen based on the Akaike information criterion (AIC) in jModeltest 2 and each alignment was analyzed using PhyML 3. In order to assess the phylogenetic position of Mariner5_Dbi in the mariner tree, we have aligned the amino acid sequence of a transposase representative of most described mariner subfamily and performed a neighbor joining analysis using MEGA 5 (JTT model, 1000 bootstrap replicates). The accession numbers of the sequences we have used are provided in Additional file 1: Figure S1.

\section{Additional files}

Additional file 1: Figure S1. Phylogenetic relationships between various mariner transposases showing that Mariner-5_Dbi belongs to the irritans subfamily.

Additional file 2: Figure S2. Illustration of the position of the two types of primer sets we used to screen for Mariner-5_Avul and Crmar2_Avul elements in the various isopod species. The sequence of the primers is provided in Additional file 5: Table S1. TIR: Terminal inverted repeat.

Additional file 3: Figure S3. Pictures of agarose gels showing the results of the reverse transcription PCR experiments on Crmar2 (A) and Mariner-5 (B) in Armadillidium vulgare ovaries and somatic tissues (head + nervous chain). A band of the expected size was obtained for all reactions showing that both elements are transcribed in A. vulgare soma and germ line. RT, reverse transcriptase; $L$, size ladder.

Additional file 4: Dataset 1. Sequences of the Crmar2 transcripts encoding a full length, intact, and thus potentially functional transposase aligned together with the Crmar2_Avul consensus sequence (fasta format).

Additional file 5: Table S1. List of primers used to amplify and sequence Crmar2 and Mariner-5 elements.

Additional file 6: Table S2. List of primers used to amplify and sequence the androgenic gland hormone.

Additional file 7: Dataset 2. Consensus sequences of Crmar2 and Mariner-5 elements reconstructed in this study (fasta format).

Additional file 8: Table S3. List of genes sequenced by Regier et al. [7] used to calculate genetic distances between the various species included in this study.

Additional file 9: Dataset 3. Sequence alignments used to reconstruct the phylogeny of crustacean isopod species and Crmar2 and Mariner-5 copies (fasta format).

\section{Abbreviations}

HTT: horizontal transfer of transposons; LTR: long terminal repeat; myr: million year; RT-PCR: reverse-transcription polymerase chain reaction; TE: transposable element.

\section{Competing interests}

The authors declare that they have no competing interests.

\section{Authors' contributions}

MD and CG designed the study, carried out the experiments and analyses and drafted the manuscript. SL generated the draft assembly of the Armadillidium vulgare genome and contributed to the writing of the manuscript. NC sequenced the androgenic gland hormone gene in the various isopod species and contributed to the writing of the manuscript. DB provided the draft transcriptome assembly of $A$. vulgare and contributed to the writing of the manuscript. All authors read and approved the final manuscript.

\section{Acknowledgements}

We thank all the technical staff of the UMR CNRS 7267. We acknowledge Catherine Debenest for assistance with the dissection of terrestrial isopods and Richard Cordaux and Pierre Grève for insightful comments on an earlier version of the manuscript. This work was supported by the CNRS, the Agence Nationale de la Recherche (ANR ImmunSymbArt 10-BLAN-1701), and a European Research Council Starting Grant (FP7/2007-2013, grant 260729 EndoSexDet) to Richard Cordaux.

\section{Author details}

${ }^{1}$ Université de Poitiers, UMR CNRS 7267 Ecologie et Biologie des Interactions, Equipe Ecologie Evolution Symbiose, 86022 Poitiers, Cedex, France. ${ }^{2}$ Present address: Courant Research Center Geobiology, Geomicrobiology and Symbiosis Group, University of Göttingen, Goldschmidtstraße 3, 37077 Göttingen, Germany.

Received: 11 October 2013 Accepted: 6 January 2014

Published: 29 January 2014

\section{References}

1. Keeling PJ, Palmer JD: Horizontal gene transfer in eukaryotic evolution. Nat Rev Genet 2008, 9:605-618.

2. Schaack S, Gilbert C, Feschotte C: Promiscuous DNA: horizontal transfer of transposable elements and why it matters for eukaryotic evolution. Trends Ecol Evol 2010, 25:537-546.

3. Ivancevic AM, Walsh AM, Kortschak RD, Adelson DL: Jumping the fine LINE between species: Horizontal transfer of transposable elements in animals catalyses genome evolution. Bioessays 2013, 35:1071-1082.

4. Wallau GL, Ortiz MF, Loreto EL: Horizontal transposon transfer in eukarya: detection, bias, and perspectives. Genome Biol Evol 2012, 4:689-699.

5. Colbourne JK, Pfrender ME, Gilbert D, Thomas WK, Tucker A, Oakley TH, Tokishita S, Aerts A, Arnold GJ, Basu MK, Bauer DJ, Cáceres CE, Carmel L, Casola C, Choi JH, Detter JC, Dong Q, Dusheyko S, Eads BD, Fröhlich T, Geiler-Samerotte KA, Gerlach D, Hatcher P, Jogdeo S, Krijgsveld J, Kriventseva EV, Kültz D, Laforsch C, Lindquist E, Lopez J, et al: The ecoresponsive genome of Daphnia pulex. Science 2011, 331:555-561.

6. Martin JW, Davis GE: An updated classification of the recent Crustacea. Science Series 39. Los Angeles, CA: Natural History Museum of Los Angeles County; 2001.

7. Regier JC, Shultz JW, Zwick A, Hussey A, Ball B, Wetzer R, Martin JW, Cunningham CW: Arthropod relationships revealed by phylogenomic analysis of nuclear protein-coding sequences. Nature 2010, 463:1079-1083.

8. Schmalfuss H: World catalog of terrestrial isopods (Isopoda: Oniscidea), Stuttgarter Beitrage zur Naturkunde Serie A, Volume Serie A, Nr. 654 Stuttgart; 2003:341

9. Broly P, Deville P, Maillet S: The origin of terrestrial isopods (Crustacea: Isopoda: Oniscidea). Evol Ecol 2013, 27:461-476.

10. Jurka J: Repbase update: a database and an electronic journal of repetitive elements. Trends Genet 2000, 16:418-420.

11. Gomulski LM, Torti C, Bonizzoni M, Moralli D, Raimondi E, Capy P, Gasperi G, Malacrida AR: A new basal subfamily of Mariner elements in Ceratitis rosa and other tephritid flies. J Mol Evol 2001, 53:597-606.

12. Kojima KK, Jurka J: DNA transposons from the Drosophila biarmipes genome. Repbase Reports 2012, 12:740.

13. Wicker T, Sabot F, Hua-Van A, Bennetzen JL, Capy P, Chalhoub B, Flavell A, Leroy P, Morgante M, Panaud O, Paux E, SanMiguel P, Schulman AH: A unified classification system for eukaryotic transposable elements. Nat Rev Genet 2007, 8:973-982. 
14. Hartl DL, Lohe AR, Lozovskaya ER: Modern thoughts on an ancyent marinere: function, evolution, regulation. Annu Rev Genet 1997, 31:337-358

15. Plasterk $\mathrm{RH}$, Izsvák Z, Ivics Z: Resident aliens: the Tc1/mariner superfamily of transposable elements. Trends Genet 1999, 15:326-332.

16. Rouault JD, Casse N, Chénais B, Hua-Van A, Filée J, Capy P: Automatic classification within families of transposable elements: application to the mariner Family. Gene 2009, 448:227-232.

17. Silva JC, Loreto EL, Clark JB: Factors that affect the horizontal transfer of transposable elements. Curr Issues Mol Biol 2004, 6:57-71.

18. Tamura K, Subramanian S, Kumar S: Temporal patterns of fruit fly (Drosophila) evolution revealed by mutation clocks. Mol Biol Evol 2004, 21:36-44.

19. Regier JC, Shultz JW, Kambic RE: Pancrustacean phylogeny: hexapods are terrestrial crustaceans and maxillopods are not monophyletic. Proc Biol Sci 2005, 272:395-401.

20. Sharkey MJ: Phylogeny and classification of hymenoptera. Zootaxa 2007, 1668:521-548

21. Hedges SB, Dudley J, Kumar S: TimeTree: a public knowledge-base of divergence times among organisms. Bioinformatics 2006, 22:2971-2972.

22. Lampe DJ, Witherspoon DJ, Soto-Adames FN, Robertson HM: Recent horizontal transfer of mellifera subfamily Mariner transposons into insect lineages representing four different orders shows that selection acts only during horizontal transfer. Mol Biol Evol 2003, 20:554-562.

23. Michel-Salzat A, Bouchon D: Phylogenetic analysis of mitochondrial LSU rRNA in oniscids. C R Acad Sci III 2000, 323:827-837.

24. Cutter AD: Divergence times in Caenorhabditis and Drosophila inferred from direct estimates of the neutral mutation rate. Mol Biol Evol 2008, 25:778-786.

25. Keightley PD, Trivedi U, Thomson M, Oliver F, Kumar S, Blaxter ML: Analysis of the genome sequences of three Drosophila melanogaster spontaneous mutation accumulation lines. Genome Res 2009, 19:1195-1201.

26. Obbard DJ, Maclennan J, Kim KW, Rambaut A, O'Grady PM, Jiggins FM: Estimating divergence dates and substitution rates in the Drosophila phylogeny. Mol Biol Evol 2012, 29:3459-3473.

27. Houck MA, Clark JB, Peterson KR, Kidwell MG: Possible horizontal transfer of Drosophila genes by the mite Proctolaelaps regalis. Science 1991, 253:1125-1128.

28. Yoshiyama M, Tu Z, Kainoh Y, Honda H, Shono T, Kimura K: Possible horizontal transfer of a transposable element from host to parasitoid. Mol Biol Evol 2001, 18:1952-1958.

29. Gilbert C, Schaack S, Pace JK II, Brindley PJ, Feschotte C: A role for hostparasite interactions in the horizontal transfer of transposons across phyla. Nature 2010, 464:1347-1350.

30. Kuraku S, Qiu H, Meyer A: Horizontal transfers of Tc1 elements between teleost fishes and their vertebrate parasites, lampreys. Genome Biol Evol 2012, 4:929-936.

31. Wijayawardena BK, Minchella DJ, DeWoody JA: Hosts, parasites, and horizontal gene transfer. Trends Parasitol 2013, 29:329-338.

32. Routh A, Domitrovic T, Johnson JE: Host RNAs, including transposons, are encapsidated by a eukaryotic single-stranded RNA virus. Proc Natl Acad Sci U S A 2012, 109:1907-1912.

33. Jehle JA, Fritsch E, Nickel A, Huber J, Backhaus H: TCl4.7: a novel lepidopteran transposon found in Cydia pomonella granulosis virus. Virology 1995, 207:369-379.

34. Jehle JA, Nickel A, Vlak JM, Backhaus H: Horizontal escape of the novel Tc1-like lepidopteran transposon TCp3.2 into Cydia pomonella granulovirus. J Mol Evol 1998, 46:215-224.

35. Piskurek O, Okada N: Poxviruses as possible vectors for horizontal transfer of retroposons from reptiles to mammals. Proc Natl Acad Sci U S A 2007, 104:12046-12051.

36. Williams T: Natural invertebrate hosts of iridoviruses (Iridoviridae). Neotrop Entomol 2008, 37:615-632.

37. Lupetti P, Montesanto G, Ciolfi S, Marri L, Gentile M, Paccagnini E, Lombardo BM: Iridovirus infection in terrestrial isopods from Sicily (Italy). Tissue Cell 2013, 45:321-327.
38. Piégu B, Guizard S, Spears T, Cruaud C, Couloux A, Bideshi DK, Federici BA Bigot Y: Complete genome sequence of invertebrate iridescent virus 22 isolated from a blackfly larva. J Gen Virol 2013, 94:2112-2116.

39. Fenn K, Conlon C, Jones M, Quail MA, Holroyd NE, Parkhill J, Blaxter M: Phylogenetic relationships of the Wolbachia of nematodes and arthropods. PLoS Pathog 2006, 2:e94.

40. Bouchon D, Cordaux R, Grève P: Feminizing Wolbachia and the evolution of sex determination in isopods. In Insect Symbiosis. 3rd edition. Edited by Bourtzis K, Miller T. Boca Raton, FL: Taylor and Francis Group LLC; 2008:273-294.

41. Coscrato VE, Braz AS, Perondini ALP, Selivon D, Marino CL: Wolbachia in Anastrepha fruit flies (Diptera: Tephritidae). Curr Microbio/ 2009, 59:295-301.

42. Cordaux R, Bouchon D, Grève P: The impact of endosymbionts on the evolution of host sex-determination mechanisms. Trends Genet 2011, 27:332-341.

43. Ravikumar H, Prakash BM, Sampathkumar S, Puttaraju HP: Molecular subgrouping of Wolbachia and bacteriophage WO infection among some Indian Drosophila species. J Genet 2011, 90:507-510.

44. Cordaux R, Michel-Salzat A, Bouchon D: Wolbachia infection in crustaceans: novel hosts and potential routes for horizontal transmission. J Evol Biol 2001, 14:237-243.

45. Klasson L, Kambris Z, Cook PE, Walker T, Sinkins SP: Horizontal gene transfer between Wolbachia and the mosquito Aedes aegypti. BMC Genomics 2009, 10:33.

46. Woolfit M, Iturbe-Ormaetxe I, McGraw EA, O'Neill SL: An ancient horizontal gene transfer between mosquito and the endosymbiotic bacterium Wolbachia pipientis. Mol Biol Evol 2009, 26:367-374.

47. Duplouy A, Iturbe-Ormaetxe I, Beatson SA, Szubert JM, Brownlie JC, McMeniman CJ, McGraw EA, Hurst GD, Charlat S, O'Neill SL, Woolfit M: Draft genome sequence of the male-killing Wolbachia strain wBol1 reveals recent horizontal gene transfers from diverse sources. BMC Genomics 2013, 14:20.

48. Robertson HM: The mariner transposable element is widespread in insects. Nature 1993, 362:241-245.

49. Robertson HM: Evolution of DNA transposons in eukaryotes. In Mobile DNA II. Edited by Craig NL, Robert Craigie R, Gellert M, Lambowitz A. Washington, DC: ASM Press; 2002:1093-1110.

50. Lorite P, Maside X, Sanllorente O, Torres MI, Periquet G, Palomeque T: The ant genomes have been invaded by several types of Mariner transposable elements. Naturwissenschaften 2012, 99:1007-1020.

51. Kidwell MG: Evolutionary biology, voyage of an ancient mariner. Nature 1993, 362:202.

52. Casse N, Bui QT, Nicolas V, Renault S, Bigot Y, Laulier M: Species sympatry and horizontal transfers of Mariner transposons in marine crustacean genomes. Mol Phylogenet Evol 2006, 40:609-619.

53. Greve P, Braquart-Varnier C, Strub JM, Felix C, Van Dorsselaer A, Martin G: The glycosylated androgenic hormone of the terrestrial isopod Porcellio scaber (Crustacea). Gen Comp Endocrinol 2004, 136:389-397.

54. Tamura K, Peterson D, Peterson N, Stecher G, Nei M, Kumar S: MEGA5: molecular evolutionary genetics analysis using maximum likelihood, evolutionary distance, and maximum parsimony methods. Mol Biol Evol 2011, 28:2731-2739

55. Hall T: BioEdit version 5.0.6. http://www.mbio.ncsu.edu/BioEdit/bioedit.html.

56. Guindon S, Gascuel O: A simple, fast, and accurate algorithm to estimate large phylogenies by maximum likelihood. Syst Biol 2003, 52:696-704.

57. Ronquist F, Teslenko M, van der Mark $P$, Ayres DL, Darling A, Höhna S, Larget B, Liu L, Suchard MA, Huelsenbeck JP: MrBayes 3.2: efficient Bayesian phylogenetic inference and model choice across a large model space. Syst Biol 2012, 61:539-542.

58. Darriba D, Taboada GL, Doallo R, Posada D: jModelTest 2: more models, new heuristics and parallel computing. Nat Methods 2012, 9:772.

\section{doi:10.1186/1759-8753-5-4}

Cite this article as: Dupeyron et al:: Horizontal transfer of transposons between and within crustaceans and insects. Mobile DNA 2014 5:4. 